\title{
Dry Slugging Granulation
}

National Cancer Institute

\section{Source}

National Cancer Institute. Dry Slugging Granulation. NCI Thesaurus. Code C113006.

The act of forming granules by mechanically applying pressure by punch and die to form a compact that is subsequently milled and screened resulting in dry granules. 